Voix et Images

voixetimages

\title{
Études françaises, vingt ans après
}

\section{Laurent Mailhot}

Volume 12, numéro 2 (35), hiver 1987

Jacques Brault

URI : https://id.erudit.org/iderudit/200636ar

DOI : https://doi.org/10.7202/200636ar

Aller au sommaire du numéro

Éditeur(s)

Université du Québec à Montréal

ISSN

0318-9201 (imprimé)

1705-933X (numérique)

Découvrir la revue

Citer cet article

Mailhot, L. (1987). Études françaises, vingt ans après. Voix et Images, 12(2),

284-287. https://doi.org/10.7202/200636ar d'utilisation que vous pouvez consulter en ligne.

https://apropos.erudit.org/fr/usagers/politique-dutilisation/ 


\section{Études françaises, vingt ans après}

\section{par Laurent Mailhot, Université de Montréal}

Malgré ce titre à la Dumas, je n'entends pas raconter des aventures rocambolesques, voire héroïques, mais faire un rapide inventaire et une sorte de bilan du passif et de l'actif - surtout de l'actif -- de la revue que je dirige. Son évolution, durant plus de vingt ans, a été marquée par des phases assez nettes, des expériences diverses et utiles. La structure et le visage actuels d'Études françaises se sont dessinés et construits peu à peu, par retouches. Après une vingtaine d'années, une revue a acquis certains traits qui sont devenus sa seconde nature, et qui la définissent plus, sinon mieux, que tous les programmes et déclarations d'intention!

Quels sont ces traits, quelle est l'image que projette notre revue auprès de ses lecteurs, auprès des critiques, auprès des autres revues, ici et à l'étranger? Nous avons peu de moyens, évidemment, de le savoir avec précision. Si lon se fie. cependant, aux rapports des divers examinateurs sollicités par les organismes subventionneurs, particulièrement par le Fonds FCAR, Etudes françaises est une revue largement mais précisément culturelle, sérieuse mais lisible, utilisable dans les classes mais peu scolaire, qui a su se donner au fil des ans une personnalité, un ton et un style assez distinctifs ${ }^{2}$.

La revue Études françaises a été fondée par le directeur du département du même nom, René de Chantal en 1965. Dans son avant-propos inaugural, celui-ci souhaitait un resserrement des liens, déjà étroits, qui unissent les universités d'Europe à celles du Canada français; il voulait, pour que les échanges se fassent dans les deux sens, initier le public d'outre-mer aux problèmes si particuliers de la littérature canadienne-française; il ouvrait aussi la porte aux professeurs canadiens-anglais et américains qui cherchent 
une tribune pour s'exprimer ${ }^{3}$. Cette porte-ci n'a jamais pu s'ouvrir aussi largement que la première, la porte transatlantique, et celle-ci n'a pas fonctionné tout à fait dans le sens prévu ou souhaité par René de Chantal. Je veux dire: nous n'avons pas échangé notre littérature canadienne-française contre la littérature française ou les littératures européennes; nous avons plutôt échangé nos idées. nos textes, sur toute la littérature, sinon sur toutes les littératures, et dans tous les sens ${ }^{4}$.

Trois phases ou étapes principales marquent l'évolution d'Êtudes françaises. De sa fondation jusqu'à 1970 , chaque numéro offre de deux à cinq articles, quelques chroniques ("Lettres françaises" et "Lettres canadiennesfrançaises"), y compris, au début, une revue d'"Actualités" ou des "Notes de lecture", ainsi qu'une bibliographie systématique des lettres canadiennesfrançaises (celle de Réginald Hamel, adaptée). Les "Notes de lecture» ou "Notes et documents" seront suivis de comptes rendus à partir de 1966. La première rupture structurelle - annonciatrice - est celle du numéro spécial sur Nelligan, en août 1967. Désormais, chaque été, un numéro thématique ("Chateaubriand et ses précurseurs français d"Amérique", etc.) viendra rompre l'uniformité des trois autres saisons, car la revue paraît alors quatre fois par année.

En février 1970, Études françaises change de couverture, élimine ses comptes rendus (remplacés par une ou plusieurs chroniques de littérature québécoise), diminue le nombre de ses "Notes et documents", qui sont d'ailleurs souvent de petits articles.

Un «texte de création" est ajouté au sommaire à partir de février 1968. Ce numéro s'ouvre sur un éditorial intitulé "Critique, création, recherche", où Georges-André Vachon, directeur depuis déjà deux ans, expose sa conception de la revue 5 . Pour lui, la "recherche" d'un poète, d'un romancier - exploration méthodique, par le recours à toutes les ressources expressives du langage, d'une réalité à découvrir - n'est pas nécessairement d'une nature différente de la recherche critique, qu'elle soit de type historique, stylistique ou thématique. Vachon insiste sur le "risque - - le beau risque - que propose le contact direct avec les textes. Il renvoie tout chercheur à la liberté du lecteur, c'est-à-dire, en un sens, du créateur. Le texte critique, selon Vachon, doit être, comme le poème ou le roman, habité par la présence d'un auteur. Ils s'adressent tous au même public, conclut-il, et ce public, ils le créent. Ils le créent, entres autres lieux, dans et par une revue.

Cette courte note de Vachon, second fondateur de la revue, en fait, inspire encore largement Ẽtudes françaises. Même lorsque le «texte de création» disparaît comme tel (à partir de février 1974), sa présence diffuse, et parfois précise, se fait sentir. L'éditorial de 1968, qui justifiait l'insertion d'un "texte de création", en annonçait déjà, par ses principes, l'extension jusqu’à un certain point à l'ensemble des articles: "Critique, création, recherche", trois mouvements en un seul.

Nous en sommes, en 1974, à la troisième phase de l'évolution d'Études françaises: changement de périodicité (trois fois l'an au lieu de quatre), nouvelle maquette, couverture en couleur et surtout numéros thématiques: 
"Écrire c'est parler», "Le bestiaire perdu», etc.. avec, pour un temps, un numéro (en mai) consacré aux chroniques et notes de lecture de l'année littéraire québécoise. Au bout de deux ans, ce numéro-panorama est abandonné et la revue devient exclusivement thématique, à une ou deux exceptions près, tels le "Petit Manuel de littérature québécoise", notre best-seller, et. signée du seul nom de Thomas A. Hale, une bibliographie commentée des Ecrits d'Aimé Césaire ${ }^{6}$, qui fait un peu bizarre - livre à part - dans notre collection.

Après cet écart bibliographique et monographique, Études françaises revient à sä thématicité. C'est le moment où le directeur actuel succède à Georges-André Vachon. On maintient encore quelque temps la tradition (établie depuis la fin de 1975) des quatre numéros sous forme de deux numéros dits «doubles", avant d'en venir, au printemps 1982, aux trois numéros annuels, tout en rêvant de leur en ajouter un jour un quatrième.

Voilà la marche - pédestre, forcément, et je m'en excuse - d'une revue en train de se faire. Sa formule, sa forme, son format ne sont sans doute pas définitivement fixés - nous songeons à ajouter bientôt une chronique de théorie littéraire couvrant ce qu'on peut appeler la "grande actualité" plutôt que l'actualité immédiate des publications - mais la revue, semble-t-il, a acquis certains traits spécifiques. Elle est identifiable par son mode de fonctionnement (en collaboration étroite avec les Presses de l'Université de Montréal), sa présentation (couverture variée, colorée, illustrations de divers types, typographie), le souci de la langue et de l'écriture, malgré des lourdeurs et des fautes inévitables. Elle est identifiable surtout par son caractère à la fois québécois et international (jusqu'au sein de son nouveau Conseil ${ }^{7}$ ). littéraire et interdisciplinaire. Études littéraires, bien sûr, partage avec nous certaines caractéristiques. Mais ici encore, si les différences sont plus subtiles, elles n’en sont pas moins réelles. Nous allons très peu du côté du Tiers-Monde et de la francophonie, domaines où l'Université Laval est mieux outillée que nous; d'autre part, nous ratissons un peu plus large - certains diront peutêtre: trop large - du côté des arts. des sciences humaines et même, à l'occasion, des sciences. Après une "Anatomie de l'écriture" à la fois neurologique. linguistique et philosophique, nous avons eu un "Texte scientifique» et, récemment, un "Cartographies" auquel ont collaboré. en humanistes, divers spécialistes, y compris un capitaine au long cours.

Nous plaçons toujours - nous voulons placer - la littérature, l'écriture, au centre de nos préoccupations. Nous évitons - nous voulons éviter - la spécialisation étroite, l'érudition lourde, les méthodologies trop scolaires. Il est bon parfois que quelques artistes - Hervé Guibert et Robert Racine, par exemple - viennent aider les historiens, les critiques d'art, les critiques littéraires, à "Êcrire l'image" - et à la lire. Une revue est un laboratoire, un atelier, ouvert non pas à tous indifféremment mais à tous ceux qui veulent $y$ travailler aux frontières de divers discours, à la jonction de la théorie et de la pratique, du texte littéraire et de ses contextes. Si ce n'est pas là, toujours, tout à fait notre histoire et notre actualité. c'est en tout cas notre programme. notre projet. 
1. Comme je l'écrivais déjà, "Quinze ans après" (Études françaises, 15: 1-2, avril 1979. p. 3). Pour une récapitulation systématique des sujets, thèmes, auteurs et collaborateurs. voir l'Index 1965-1984, publié en 1986 (77 p.) aux PUM.

2. A titre d'exemples, voici quelques commentaires des appréciateurs externes lors de l'évaluation comparative du Fonds FCAR en 1985: Etudes françaises a choisi de privilégier les problèmes théoriques que pose la production littéraire. Ce choix peut se défendre, d'autant plas qu'elle est la seule revue à avoir agi de la sorte. du moins de façon aussi systénatique; La force d'Études françaises réside dans sa manière dexplorer et de maitriver le's grandes interrogations théoriques que posé la produrtion litréraire. La lecture des numéros que vous miave a soumis est particulièrement fécomder.

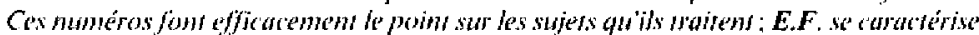
par la qualire de sa reflexion et de son écriture: elle est proprentent une rovere de

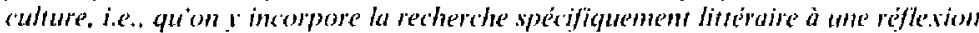

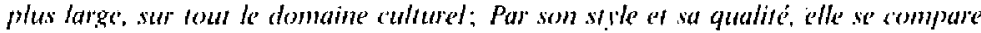

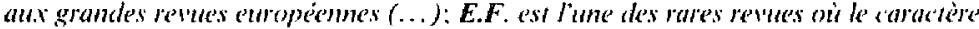

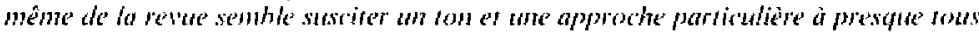

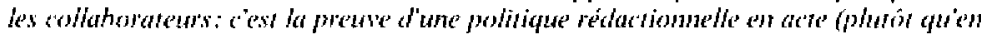
principe)

3. 1: I. février 1965.

4. Presque tous les numéros récents. même ceux qui portent sur des écrivains québécois ( VL.B", "lacques Poulin", "Voyages en Nouvelle-France»), comprennent plusicurs articles d'origine européenne et américaine.

5. Voir alussi "Faire la littérature» (6: 1, février $[970$, p. 3-6).

6. 14:3-4 octobre 1978 .

7. Créé en 1984. 